\title{
Comparison of Two Tube-Modification Techniques in Baerveldt Glaucoma Implantation in Refractory Glaucoma
}

This article was published in the following Dove Press journal: Clinical Ophthalmology

\author{
Purit Petpiroon' \\ Thanyathorn \\ Vijittrakarnrung (iD) \\ Wasu Supakontanasan' \\ Apichat Tantraworasin (D) ${ }^{2,3}$ \\ Yanin Suwan (iD) \\ 'Department of Ophthalmology, Faculty \\ of Medicine, Ramathibodi Hospital, \\ Mahidol University, Bangkok, Thailand; \\ ${ }^{2}$ Department of Surgery and Clinical \\ Epidemiology and Clinical Statistic \\ Center, Faculty of Medicine, Chiang Mai \\ University, Chiang Mai, Thailand; \\ ${ }^{3}$ Pharmacoepidemiology and Statistics \\ Research Center (PESRC), Faculty of \\ Pharmacy, Chiang Mai University, Chiang \\ Mai, Thailand
}

Correspondence: Yanin Suwan

Department of Ophthalmology, Faculty of Medicine, Ramathibodi Hospital, Mahidol University, Bangkok, Thailand

Tel +662-20l-2729

Fax +662-354-7280

Email yanin.suwan@gmail.com
Importance: To compare treatment outcomes among a stent group, ligature group and combined group in eyes with refractory glaucoma.

Background: Various techniques have been used intra-operatively to restrict the aqueous flow in Baerveldt glaucoma implantation.

Design: Retrospective chart review.

Participants: All glaucoma patients aged over 18-years old who had Baerveldt implantation in Ramathibodi Hospital, Bangkok, Thailand, between October 2011 and February 2018 were included for analysis.

Methods: Retrospective interventional research was conducted. All glaucoma patients who underwent drainage device implantations were retrospectively reviewed from medical records and divided into three groups: stent group, ligature group, and combined group. The primary outcome was post-operative intraocular pressure (IOP) changes and BCVA $(\log M A R)$ change. The secondary outcome is treatment failure. Repeated measurements with mixed models and multi-level parametric survival model stratified by propensity score and eye side were used to compare the primary and secondary outcomes between stent group and ligature group.

Main Outcome Measures: Treatment failure between two groups.

Results: A total of 163 patients with a mean age of $57.11 \pm 19.04$ years, implanted with Baerveldt tube, were eligible. There were no significant differences between stent and ligature groups in terms of post-operative IOP changes [mean difference with $95 \%$ confidence interval $=0.53(-0.49,+1.55)$ vs $-0.02(-0.84,+0.81) ; \mathrm{P}=0.411]$ and post-operative BCVA (logMAR) change $(0.02(-0.13,+0.18)$ vs $-0.05(-0.18,+0.07) ; \mathrm{P}=0.465)$. The hazard ratio and $95 \%$ confidence interval of treatment failure in ligature group were 2.57 (0.72-9.19) compared with the stent group.

Conclusion: This study suggests a trend toward a better result in the stent group compared with the ligature group. Further research with a larger sample size and randomized control trial is warranted.

Keywords: glaucoma drainage device, tube shunt, glaucoma surgery, Baerveldt glaucoma implantation

\section{Introduction}

Glaucoma refractory to maximum tolerated medical therapy and laser trabeculoplasty often requires surgical intervention to lower intraocular pressure (IOP) and prevent ultimate vision loss. The most commonly performed procedure is trabeculectomy, which drains the aqueous through an artificial fistulous tract and creates a bleb. 
However, high risks of bleb-related complications and failure indicate that the use of glaucoma drainage devices (GDDs) may be a preferable alternative procedure. ${ }^{1,2}$ The general purpose of these implants is to divert aqueous to the equatorial region through permanent sclerotomy (tube). GDDs are increasingly utilized in the management of refractory glaucoma. $^{3-6}$

In the past 2 decades, several types of glaucoma drainage implants have been developed. One of the most commonly used shunts is a non-valved Baerveldt Glaucoma Implant (BGI). Previous studies have concluded that the BGI is more effective than Ahmed glaucoma valves both in terms of surgical success rate and ability to reduce glaucoma medication..$^{7-9}$ Because BGI does not have a flow restrictor, hypotony and its associated complications are theoretically more common. ${ }^{6,10,11}$ One of the biggest challenges of nonvalved drainage device surgery is the prevention of hypotony during the early post-operative period.

Method wherein aqueous flow is blocked to form capsule to prevent further hypotony include ligation (tying a suture around the implant tube), stenting (threading a suture into the tube lumen) and their combination. ${ }^{6,12-16}$ Limited data exist between stenting and ligation. Therefore, this study compared treatment outcomes in eyes with refractory glaucoma: stent group, ligature group and combined group.

\section{Patients and Methods}

In this study, the medical records of all patients who underwent BGI at Ramathibodi Hospital (Mahidol University, Bangkok, Thailand) between October 2011 and February 2018 were retrospectively reviewed. The study protocol was approved by the institutional review board of the Hospital and adhered to the tenets of the Declaration of Helsinki. The medical records of patients with refractory glaucoma aged over 18 years old who underwent BGI were included. Refractory glaucoma was defined as patient with uncontrolled intraocular pressure more than $21 \mathrm{mmHg}$ despite maximal antiglaucoma medication, previously failed non-seton surgical treatment, or a combination. Data collected included details of the surgical procedure. Data related to best-corrected visual acuity (BCVA) in logMAR unit, IOP and IOP-lowering medications at each follow-up were also recorded.

\section{Surgical Technique}

All BGIs were performed by one of the three glaucoma attending physicians or glaucoma fellows under direct supervision. Conjunctival peritomy was performed at an angle of $100-120^{\circ}$ with radial incision. Two rectus muscles were identified and secured by silk $2-0$. The patency of the Baerveldt tube was confirmed by fluid irrigation before implantation. Then, the device was placed and fixed to the underlying sclera $10 \mathrm{~mm}$ away from the limbus. No antimetabolites were used intra-operatively. For aqueous blockage, ligation (7-0 polyglactin $\left[\right.$ Vicryl $\left.{ }^{\circledR}\right]$ ), stenting (4-0 chromic catgut) or their combined technique (ligation with 7-0 polyglactin and stenting with 4-0 chromic catgut) was used, depending on the surgeon's preference. Further, $2-3$ venting slits were created proximally to the ligated site. ${ }^{17}$ The tube was trimmed to an appropriate length and inserted into the anterior chamber, ciliary sulcus or pars plana under the scleral flap and preserved scleral graft with the bevel facing anteriorly through a 23 -gauge needle track. The conjunctiva was closed with a 7-0 polyglactin suture. Patients were administered a combination of a topical antibiotic and steroid four times daily, the dose of which was then tapered over 2 months. Chromic catgut suture was removed at post-operative $4-6$ weeks. Once conjunctival wound completely healed and anterior chamber inflammation subsided, chronic catgut suture could be removed. Polyglactin suture was retained to be spontaneously absorbed without adjunctive laser suture lysis.

\section{Outcome Measurements}

Treatment failure was defined as an IOP $\geq 21 \mathrm{mmHg}$ on $\geq 2$ consecutive follow-up visits; an IOP lowering of $<20 \%$ from baseline, despite the use of IOP-lowering medications; need for further surgical intervention; or no light perception of the visual acuity.

\section{Statistical Analysis}

Statistical analysis was performed with Stata (ver. 15.1, StataCorp, College Station, Texas, USA). Data are presented as mean \pm standard deviation, unless stated otherwise, and compared among three groups using one-way ANOVA or Kruskal-Wallis test depending on data distribution. Categorical data are presented as frequency (percent) and compared among three groups using chi-square test or fisher exact probability test as appropriate. In multivariable analysis to compare the primary and secondary outcome, combined group was excluded for data analysis because of small sample size. The outcomes of treatment were compared between ligature group and stent group. Logistic regression was used to calculate a propensity score (PS), which evaluates confounding by indication. The variables included in the 
model for PS were age, gender, eye side, diagnosis, previous surgery, lens status, preoperative BCVA (logMAR), preoperative IOP, implant location, tube fixation and Baerveldt model. Repeated measurements with mixed models stratified by PS and eye side was used for comparing the primary outcomes demonstrated with a mean difference with $95 \%$ confidence interval (CI). A multilevel parametric survival analysis model stratified by PS and eye side was used for comparing the secondary outcome (treatment failure) between groups and demonstrated with a hazard ratio (HR) with $95 \%$ CI. The cumulative hazard function of treatment failure analyzed by mixed-effects exponential regression was demonstrated. Cut off for statistical significance was set at $\mathrm{P}<0.05$.

\section{Results}

In total, 174 eyes (165 patients) implanted with BGI were eligible. Among these, 163 were primary implants and 11 were sequential implants. Nine patients with both eyes were eligible. This study included only primary GDDs (ie primary BGI); sequential GDDs were excluded. Demographic characteristics of patients are shown in Table 1.

Table 1 summarizes the baseline characteristics of the study sample. Their mean patient age at the time of surgery was $57.11 \pm 19.04$ years (range: 18 to 92 years).

Demographic characteristics of patients in the stent, ligature and combined groups were compared (Table 2). There was no significant difference among the three groups in terms of diagnosis, phakic status, number of previous surgeries, pre-operative visual acuity, pre-operative IOP, implant location and location of tube fixated. However, there were significant differences among the three groups in terms of the number of pre-operative medications (stent, $4.48 \pm 0.77$; ligature, $4.07 \pm 0.63$ and combined groups, $3.67 \pm 0.52 ; \mathrm{P}=$ 0.002) and BGI model (Baerveldt 350: $18 \pm 60.00$ vs $116 \pm$ 92.06 vs $2 \pm 33.33$ and Baervedlt 250: $12 \pm 40.00$ vs $10 \pm$ 7.94 vs $4 \pm 66.67$ for the stent, ligature and combined groups, respectively; $\mathrm{P}<0.001$; Table 2). Further, 53 (32.52\%) eyes

Table I Patient Characteristics $(\mathrm{N}=154$, Eye Side $=163)$

\begin{tabular}{|l|l|}
\hline Variables & N (\%) or Mean \pm SD \\
\hline Sex & \\
Male & $88(57.14)$ \\
Female & $66(42.86)$ \\
\hline Age (years) & $57.11 \pm 19.04$ (range 18-92) \\
\hline
\end{tabular}

were diagnosed with primary open-angle glaucoma, 121 (74.23\%) had previously undergone trabeculectomy and 132 (81.48\%) had previously undergone cataract surgery. The median number of previous intraocular surgeries was 3, 3 and 2 in the stent, ligature and combined groups, respectively. The majority of eyes' phakic status was pseudophakia $(64.52 \%, 67.20 \%$ and $50.00 \%$ in the stent, ligature and combined groups, respectively). The median pre-operative best-corrected VA (BCVA) in logMAR unit was $0.88(0.30$ $1.30), 0.88(0.40-2.12)$ and $1.3(0.40-2.30)$ and mean preoperative IOP was $23.19 \pm 11.84,24.91 \pm 9.04$ and $22.67 \pm$ 6.41 in the stent, ligature and combined groups, respectively. Considering quadrant, devices were implanted superotemporally $(113,69.33 \%)$, superonasally $(21,12.88 \%)$, inferotemporally $(15,9.20 \%)$ and inferonasally.

The median (IQR) follow-up period was 60.8 (21.23-66.03), $17.63(10.23-32.33)$ and 13.1 (12.33-14.33) months for the stent, ligature and combined groups, respectively (Table 3 ).

The propensity score was calculated based on age, sex, eye side, diagnosis, number of previous surgery, lens status, pre-operative BCVA (logMAR), pre-operative IOP, implant location, location of tube fixation and BGI model

There were no significant differences between stent and ligature groups in terms of post-operative IOP changes [mean difference $(95 \%$ confidence interval $)=0.53(-0.49$, $+1.55)$ vs $-0.02(-0.84,+0.81) ; \mathrm{P}=0.411]$, analyzed by repeated measurements with mixed models stratified by propensity score and eye side (Figure 1A).

There were no significant differences between stent and ligature groups in terms of post-operative BCVA $(\log M A R)$ change [mean difference $(95 \%$ confidence interval $)=0.02$ $(-0.13,+0.18)$ vs $-0.05(-0.18,+0.07) ; \mathrm{P}=0.465]$, analyzed by repeated measurements with mixed models stratified by propensity score and eye side (Figure 1B).

The hazard ratio and 95\% confidence interval of treatment failure for the ligature group were 2.57 (0.72-9.19) compared with the stent group, analyzed using multilevel parametric survival model, stratified by eye side and propensity score, and adjusted by age, gender, previous surgery and pre-operative IOP (Figure 2). Repeated measurement analysis with excluded missing data at baseline and last follow up is available in the Supplementary material (Tables S1-S3).

\section{Discussion}

Trabeculectomy with mitomycin $\mathrm{C}$ can be effective in lowering IOP, but extensive dissection, ostomy formation and suturing can make treatment outcomes unpredictable and 
Table 2 Characteristics of the Three Groups

\begin{tabular}{|c|c|c|c|c|c|}
\hline & Total N (\%) & Stent Group $(\mathbf{N}=3 \mathrm{I})$ & Ligature Group $(n=126)$ & Combined Group $(\mathrm{N}=6)$ & p-value \\
\hline Eye side, n (\%) & $163(100)$ & & & & 0.393 \\
\hline Right (OD) & $87(53.37)$ & $16(51.61)$ & $66(52.38)$ & $5(83.33)$ & \\
\hline Diagnosis & $163(100)$ & & & & 0.321 \\
\hline POAG & $53(32.52)$ & 7 (22.58) & $43(34.13)$ & $3(50.00)$ & \\
\hline Others & $110(67.48)$ & $24(77.42)$ & $83(65.87)$ & $3(50.00)$ & \\
\hline Number of previous surgery, Median (IQR) & $160(98.16)$ & $3(2-4)$ & $3(2-3)$ & $2(2-3)$ & 0.282 \\
\hline Phakic status & $162(99.39)$ & & & & 0.312 \\
\hline Pseudophakia & $107(66.05)$ & $20(64.52)$ & $84(67.20)$ & $3(50.00)$ & \\
\hline Phakia & $30(18.52)$ & $7(22.58)$ & $20(16.00)$ & $3(50.00)$ & \\
\hline Aphakia & $25(15.43)$ & $4(12.90)$ & $21(16.80)$ & 0 & \\
\hline Preoperative LogMAR BCVA, median (IQR) & $163(100)$ & $0.88(0.30-1.30)$ & $0.88(0.40-2.12)$ & $1.3(0.40-2.30)$ & 0.776 \\
\hline Pre-operative IOP, mean \pm SD & 161 (98.77) & $23.19 \pm 11.84$ & $24.91 \pm 9.04$ & $22.67 \pm 6.41$ & 0.599 \\
\hline Pre-operative number of medications & $163(100)$ & $4.48 \pm 0.77$ & $4.07 \pm 0.63$ & $3.67 \pm 0.52$ & 0.002 \\
\hline Implant location & $163(100)$ & & & & 0.098 \\
\hline Superonasal & $21(12.88)$ & $6(19.35)$ & $15(11.90)$ & 0 & \\
\hline Superotemporal & $113(69.33)$ & $24(77.42)$ & $85(67.46)$ & $4(66.67)$ & \\
\hline Inferonasal & 14 (8.59) & I (3.23) & $13(10.32)$ & 0 & \\
\hline Inferotemporal & $15(9.20)$ & 0 & $13(10.32)$ & $2(33.33)$ & \\
\hline Tube fixation & $163(100)$ & & & & 0.836 \\
\hline Angle & $144(88.34)$ & $27(87.10)$ & $111(88.10)$ & $6(100)$ & \\
\hline Sulcus & $16(9.82)$ & $3(9.68)$ & $13(10.32)$ & 0 & \\
\hline Pars plana & $3(1.84)$ & I (3.23) & $2(1.59)$ & 0 & \\
\hline Baerveldt glaucoma implant model & $162(99.39)$ & & & & $<0.001$ \\
\hline 350 & $136(83.95)$ & $18(60.00)$ & $116(92.06)$ & $2(33.33)$ & \\
\hline 250 & $26(16.05)$ & $12(40.00)$ & $10(7.94)$ & $4(66.67)$ & \\
\hline
\end{tabular}

Note: BCVA: comparison among the three groups of tube modification using ANOVA.

Abbreviations: POAG, primary open-angle glaucoma; BCVA, best-corrected visual acuity; OD, oculus dexter; IQR, interquartile range; SD, standard deviation.

lead to complications. Common trabeculectomy complications include hemorrhage, scarring, cataract formation, hypotony, bleb-related infection, functional vision loss and failure. $^{2,18}$ Among all options, BGI is more resistant to conjunctival scarring and is an increasingly used intervention in refractory glaucoma. This device has previously shown promising implications as the first-line treatment of glaucoma in patients who have undergone intraocular surgery prior to filtering surgery. However, BGI, which is a non-valved implant, may have dreadful complications during both the early and late post-operative periods, which include post-operative hypotony/hypertony, flat anterior chamber with or without tube-endothelial touch and choroidal detachment. ${ }^{19}$ Many complications result from inappropriate aqueous flow restriction during the early postoperative period. Bleb resistance surrounding the BGI endplate tends to have minimal resistance to aqueous outflow until approximately post-operative 6-weeks. ${ }^{6}$ Various techniques have been used intra-operatively to restrict the aqueous flow in this non-valved device. External ligation, internal stenting or their combined technique has been used to achieve the optimum aqueous outflow and to allow encapsulation to form above the BGI endplate. This study found that the ability to control IOP and BCVA (logMAR) during the post-operative period were similar in both techniques. The hazard ratio and $95 \%$ confidence interval of treatment failure for the ligature group were 2.57 (95\% CI, 0.72-9.19) compared with that of the stent group at the 5 -year follow-up. There was no statistical significant difference between the two groups; however, there was a trend toward better surgical result in the stent group compared with the ligature group. One possible explanation for this is the differences in the types of absorbable sutures used. Polyglactin sutures hold their tensile strength for approximately 2-3 weeks in tissues and are completely absorbed by hydrolysis within 56-70 days [Coated VICRYL ${ }^{\circledR}$ (polyglactin 910) Violet braided absorbable suture; Ethicon Inc.]. In contrast, chromic catgut holds its tensile strength 
Table 3 Follow-Up Period and Treatment Outcomes

\begin{tabular}{|c|c|c|c|c|}
\hline Variables & Stent Group $(\mathbf{N}=31)$ & Ligature Group $(n=126)$ & Combined Group $(N=6)$ & P-value \\
\hline \multicolumn{5}{|l|}{ BCVA (logMAR), median (IQR) } \\
\hline I month $(\mathrm{N}=155)$ & $1.15(0.60-2.30)$ & $1.15(0.5 \mathrm{I}-2.60)$ & $1.23(0.60-2.90)$ & 0.989 \\
\hline 6 months $(\mathrm{N}=133)$ & $1.00(0.66-2.30)$ & $1.00(0.46-2.60)$ & $0.88(0.40-1.30)$ & 0.799 \\
\hline 12 months $(\mathrm{N}=103)$ & $0.95(0.48-2.30)$ & $0.90(0.48-2.00)$ & - & 0.888 \\
\hline 36 months $(N=42)$ & $1.00(0.40-2.60)$ & $1.00(0.62-3.00)$ & - & 0.534 \\
\hline 60 months $(\mathrm{N}=1 \mathrm{I})$ & $1.91(0.37-2.90)$ & $0.88(0.88-0.88)$ & - & 0.526 \\
\hline \multicolumn{5}{|l|}{ IOP, median (IQR) } \\
\hline I month $(N=156)$ & $16.00(11.00-22.00)$ & $13.50(10.00-18.00)$ & $16.50(10.00-19.00)$ & 0.151 \\
\hline 6 months $(N=132)$ & $12.00(9.00-15.00)$ & $14.00(10.00-17.00)$ & $15.00(12.00-20.00)$ & 0.250 \\
\hline 12 months $(\mathrm{N}=101)$ & $13.50(11.00-16.00)$ & $12.00(10.00-17.00)$ & - & 0.468 \\
\hline 36 months $(\mathrm{N}=39)$ & $12.00(12.00-15.00)$ & $14.00(12.00-17.00)$ & - & 0.393 \\
\hline 60 months $(\mathrm{N}=1 \mathrm{I})$ & $19.50(12.00-25.00)$ & $23.00(23.00-23.00)$ & - & 0.527 \\
\hline \multicolumn{5}{|l|}{ Follow-up period (months) } \\
\hline Median (IQR) & $60.68(21.23-66.03)$ & I7.63 (I0.23-32.33) & $13.1(12.33-14.33)$ & $<0.001$ \\
\hline Treatment failure & $8(26.67)$ & $29(23.58)$ & I (I6.67) & 0.348 \\
\hline \multicolumn{5}{|l|}{ Failure time (months) } \\
\hline Median (IQR) & $19.17(6.67-55.17)$ & $5.47(3.33-12.03)$ & I & 0.097 \\
\hline
\end{tabular}

Abbreviations: BCVA, best-corrected visual acuity; POAG, primary open-angle glaucoma.

for 18-21 days and is degraded by proteolytic enzymes and phagocytic cells within 90 days. Hydrolysis produces less tissue reaction than enzymatic degradation. ${ }^{20}$ Chromic catgut is a virtually monofilament suture that theoretically produces few tissue reactions than multifilament polyglactin and generates a milder inflammatory reaction at the capsular endplate level. This may lead to low drainage resistance of encapsulation. However, the in vivo inflammatory reaction between the chromic catgut and polyglactin sutures remains unclear. ${ }^{21,22}$

Despite the trend toward the better result for the stent group, chromic catgut sutures must be handled carefully because it can easily weaken or break when crushed by certain instruments.
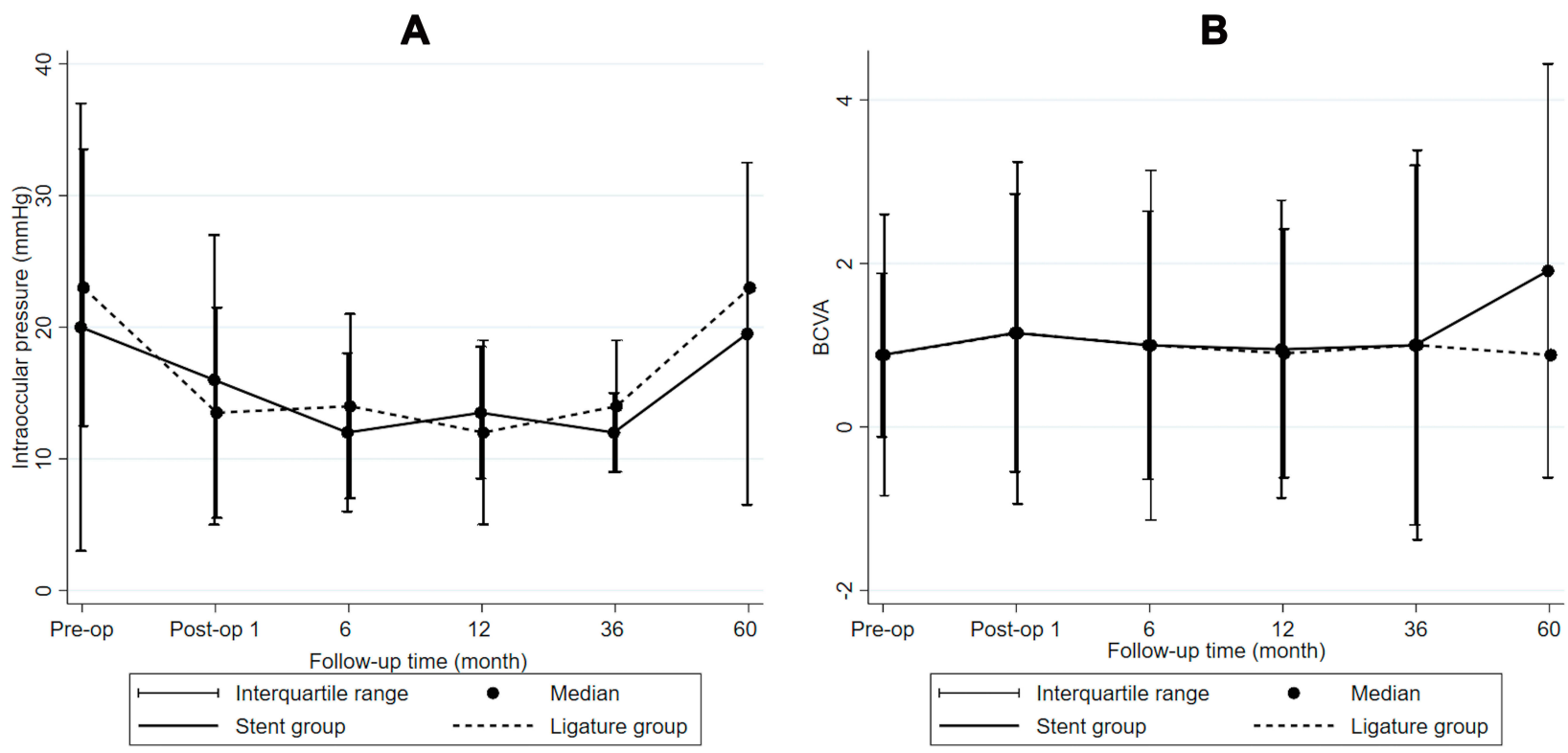

Figure I Postoperative follow-up time of IOP (A) and BCVA (logMAR) (B) between two groups. 


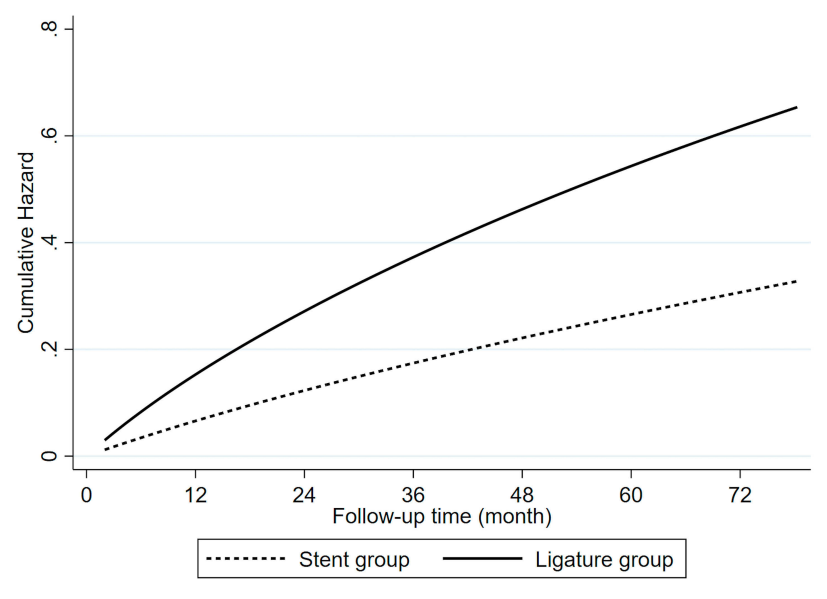

Figure 2 Adjusted parametric cumulative hazard function illustrating treatment failure comparing between the two groups and analysed by mixed-effects exponential proportional hazard regression $(P=0.147)$.

The second possible explanation for the discrepancy in the results is the difference in the timing of suture removal between the two groups. In the stent group, the chromic catgut sutures were removed at post-operative 4-6 weeks. In contrast, polyglactin suture in the ligature group was allowed to spontaneously resolve, which may vary upon individual tissue reactions.

There are some limitations to this study. First, the retrospective nature of the study may have led to significant biases. Second, the relatively small number of patients in the stent group limited the statistical power of this study to identify the surgical risk factors. Future study with larger sample size may be warranted. Third, this study included eyes that underwent multiple previous ocular surgeries. Patients were enrolled from Ramathibodi Hospital, a referral center, representing the severe end of the glaucoma spectrum. Extensive conjunctival scarring may compromise the surgical results compared with primary surgery. Finally, this study included data pertaining to drainage devices implanted by multiple surgeons using a standardized surgical technique.

To the best of our knowledge, this is the first study to compare two tube-modification techniques. This study found a trend toward a better result for the stent group than for the ligature group. Further research with large sample size and randomized control trial is warranted to confirm results and identify the risk factors associated with surgical failure.

\section{Data Sharing Statement}

The data have not been placed in any online data storage. The datasets generated and analyzed during the study are available upon request from the first author.

\section{Ethics Approval and Consent to Participate}

This study followed the tenets of the Declaration of Helsinki and was approved by the ethics committee of the Ramathibodi Hospital, Mahidol University. Written informed consent was obtained from all participants.

\section{Author Contributions}

All authors made substantial contributions to conception and design, acquisition of data, or analysis and interpretation of data; took part in drafting the article or revising it critically for important intellectual content; gave final approval of the version to be published; and agree to be accountable for all aspects of the work.

\section{Funding}

None.

\section{Disclosure}

The authors report no conflicts of interest in this work.

\section{References}

1. Leeungurasatien T, Khunsongkiet P, Pathanapitoon K, Wiwatwongwana D. Incidence of short-term complications and associated factors after primary trabeculectomy in Chiang Mai University Hospital. Indian J Ophthalmol. 2016;64:737-742.

2. Zahid S, Musch DC, Niziol LM, Lichter PR; Collaborative Initial Glaucoma Treatment Study G. Risk of endophthalmitis and other long-term complications of trabeculectomy in the Collaborative Initial Glaucoma Treatment Study (CIGTS). Am J Ophthalmol. 2013;155(4):674-80, 80 e1. doi:10.1016/j.ajo.2012.10.017

3. Roy S, Ravinet E, Mermoud A. Baerveldt implant in refractory glaucoma: long-term results and factors influencing outcome. Int Ophthalmol. 2001;24(2):93-100. doi:10.1023/A:101633531 3035

4. Souza C, Tran DH, Loman J, Law SK, Coleman AL, Caprioli J. Longterm outcomes of Ahmed glaucoma valve implantation in refractory glaucomas. Am J Ophthalmol. 2007;144(6):893-900. doi:10.1016/j. ajo.2007.07.035

5. Ko SJ, Hwang YH, Ahn SI, Kim HK. Surgical outcomes of additional Ahmed glaucoma valve implantation in refractory glaucoma. $J$ Glaucoma. 2016;25(6):e620-e624. doi:10.1097/IJG.000000000 0000298

6. Lim KS. Control and optimisation of fluid flow in glaucoma drainage device surgery. Eye (Lond). 2018;32(2):230-234. doi:10.1038/ eye.2017.316

7. Wang S, Gao X, Qian N. The Ahmed shunt versus the Baerveldt shunt for refractory glaucoma: a meta-analysis. BMC Ophthalmol. 2016;16 (1):83. doi:10.1186/s12886-016-0265-6

8. Budenz DL, Barton K, Gedde SJ, et al. Five-year treatment outcomes in the Ahmed Baerveldt comparison study. Ophthalmology. 2015;122 (2):308-316. doi:10.1016/j.ophtha.2014.08.043

9. Christakis PG, Zhang D, Budenz DL, et al. Five-year pooled data analysis of the Ahmed Baerveldt Comparison Study and the Ahmed Versus Baerveldt Study. Am J Ophthalmol. 2017;176:118-126. doi:10.1016/j.ajo.2017.01.003 
10. Law SK, Kalenak JW, Connor TB Jr, Pulido JS, Han DP, Mieler WF. Retinal complications after aqueous shunt surgical procedures for glaucoma. Arch Ophthalmol. 1996;114:1473-1480. doi:10.1001/ archopht.1996.01100140671004

11. Siegner SW, Netland PA, Urban RC Jr., et al. Clinical experience with the Baerveldt glaucoma drainage implant. Ophthalmology. 1995;102(9):1298-1307. doi:10.1016/S0161-6420(95)30871-8

12. Rose GE, Lavin MJ, Hitchings RA. Silicone tubes in glaucoma surgery: the effect of technical modifications on early postoperative intraocular pressures and complications. Eye (Lond). 1989;3(Pt 5):553-561. doi:10.1038/eye.1989.87

13. Trible JR, Brown DB. Occlusive ligature and standardized fenestration of a Baerveldt tube with and without antimetabolites for early postoperative intraocular pressure control. Ophthalmology. 1998;105:2243-2250. doi:10.1016/S0161-6420(98)91223-4

14. Molteno AC, Polkinghorne PJ, Bowbyes JA. The vicryl tie technique for inserting a draining implant in the treatment of secondary glaucoma. Aust N Z J Ophthalmol. 1986;14:343-354. doi:10.1111/ j.1442-9071.1986.tb00470.x

15. Sherwood MB, Smith MF. Prevention of early hypotony associated with Molteno implants by a new occluding stent technique. Ophthalmology. 1993;100:85-90. doi:10.1016/S0161-6420(93) 31688-X

16. Breckenridge RR, Bartholomew LR, Crosson CE, Kent AR. Outflow resistance of the Baerveldt glaucoma drainage implant and modifications for early postoperative intraocular pressure control. J Glaucoma. 2004;13:396-399. doi:10.1097/01.ijg.0000131759.48295.5c
17. Emerick GT, Gedde SJ, Budenz DL. Tube fenestrations in Baerveldt glaucoma implant surgery: 1-year results compared with standard implant surgery. J Glaucoma. 2002;11(4):340-346. doi:10.1097/ 00061198-200208000-00011

18. Levinson JD, Giangiacomo AL, Beck AD, et al. Glaucoma drainage devices: risk of exposure and infection. Am J Ophthalmol. 2015;160 (3):516-21 e2. doi:10.1016/j.ajo.2015.05.025

19. Pitukcheewanont O, Tantisevi V, Chansangpetch S, Rojanapongpun P. Factors related to hypertensive phase after glaucoma drainage device implantation. Clin Ophthalmol. 2018;12:1479-1486. doi:10.2147/ OPTH.S166244

20. Byrne M, Aly A. The surgical suture. Aesthet Surg J. 2019;39:S67S72. doi:10.1093/asj/sjz036

21. Guyuron B, Vaughan C. Comparison of polydioxanone and polyglactin 910 in intradermal repair. Plast Reconstr Surg. 1996;98 (5):817-820. doi:10.1097/00006534-199610000-00010

22. Sanz LE, Patterson JA, Kamath R, Willett G, Ahmed SW, Butterfield AB. Comparison of maxon suture with vicryl, chromic catgut, and PDS sutures in fascial closure in rats. Obstet Gynecol. 1988;71(3 Pt $1): 418-422$.
Clinical Ophthalmology

\section{Publish your work in this journal}

Clinical Ophthalmology is an international, peer-reviewed journal covering all subspecialties within ophthalmology. Key topics include: Optometry; Visual science; Pharmacology and drug therapy in eye diseases; Basic Sciences; Primary and Secondary eye care; Patient Safety and Quality of Care Improvements. This journal is indexed on PubMed
Dovepress

Central and CAS, and is the official journal of The Society of Clinical Ophthalmology (SCO). The manuscript management system is completely online and includes a very quick and fair peer-review system, which is all easy to use. Visit http://www.dovepress.com/ testimonials.php to read real quotes from published authors. 